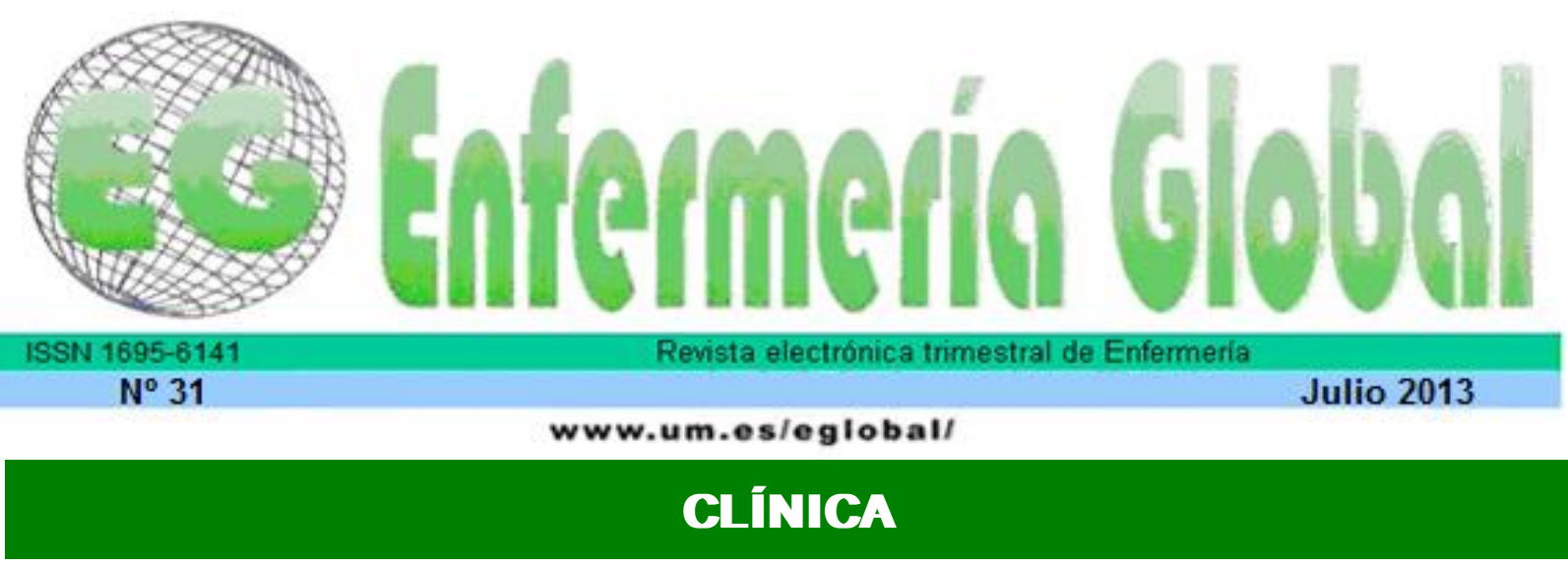

\title{
Barreras percibidas y nivel de actividad física en adultos mayores de Aguascalientes, Ags.: Un estudio transversal
}

Perceived barriers and physical activity level in older adults from Aguascalientes, Ags.: un studio transversal

\section{${ }^{*}$ Andrade Osorio, Érika ${ }^{* *}$ Padilla Raygoza, Nicolás ${ }^{* * *}$ Ruiz Paloalto, $\mathrm{M}^{a}$ Laura}

*Coordinador de la práctica de enfermería de trabajo de parto, Universidad Autónoma de Aguasaclientes. **Departamento de Enfermería y Obstetricia, División de Ciencias de la Salud e Ingenierías, Campus Celaya Salvatierra. E-mail: raygosan@ugto.mx ***Departamento de Enfermería Clínica, División de Ciencias de la Salud e Ingenierías, Campus Celaya Salvatierra. Universidad de Guanajuato. México.

Palabras clave: Adultos mayores; actividad física; barreras

.Keywords: Elderly; older adults; physical activity; barriers

\section{RESUMEN}

Introducción. México está en transición demográfica con más del 10\% de su población, siendo adultos mayores, quienes tienen elevada prevalencia de enfermedades no transmisibles. El objetivo fue conocer la relación entre las barreras percibidas y el nivel de actividad física en adultos mayores de Aguascalientes, Ags.

Materiales y métodos. Es un estudio transversal, observacional, analítico. 150 adultos mayores registrados en el Sistema de Desarrollo Integral Familiar de Aguascalientes, que firmaron el consentimiento, fueron incluidos. Se aplicaron los cuestionarios de barreras percibidas y el internacional de actividad física.

Análisis estadístico. Z para dos proporciones, valor de $\mathrm{p}$, Razón de Momios e intervalos de confianza al 95\% fueron calculados, entre barreras percibidas externas e internas y el nivel de actividad física que realizaban los adultos mayores de la muestra.

Resultados. La muestra estuvo integrada por adultos mayores con edad entre los 60 y 70 años; $72 \%$ fueron mujeres, $78 \%$ tenían bajo nivel de actividad física. La falta de información acerca de los beneficios de la actividad física es una barrera para tener mejor nivel de actividad física $(Z=2.36$, $\mathrm{p}=0.02, \mathrm{RM}=2.97 \mathrm{IC95} \%=1.28$ a 6.90 ); la falta de apoyo de las familias es otra barrera para la actividad física $(Z=2.91, p=0.003, R M=3.82$, IC95\%=161 a 9.10); también fue una barrera la falta de espacios 
para realizar actividad física, tales como centros de salud y gimnasios para adultos mayores $(Z=4.2$, $\mathrm{p}<0.05, \mathrm{RM}=5.8, \mathrm{IC} 95=2.5$ a 13.3).

Conclusión. El diseño de programas de enfermería para realizar actividad física en adultos mayors, deberá tomar en cuenta las barreras percibidas.

\section{ABSTRACT}

Introduction. Mexico is in demographic transition being more than $10 \%$ of its population older adults who have a high frequency of non-communicable diseases. The objective was to know the relationship between perceived barriers and physical activity level in older adults from Aguascalientes in Mexico.

Material and methods. The present study is cross-sectional, analytic, and observational.150 older adults registered in the Family Integral Development System from Aguascalientes State were included at random, who accepted to participate by signing a consent form. A questionnaire to detect perceived barriers as well as an international physical activity questionnaire was applied.

Statistical analysis. Z for two proportions, p-value and odds ratio, $95 \%$ confidence interval were calculated, between external and internal perceived barriers and low physical activity level in older adults from the sample.

Results. In reference to the sample, $71.3 \%$ were between 60 and 70 years old; $72 \%$ were female, $78 \%$ had low physical activity level. The lack of information about physical activity benefits is a barrier in relation to low physical activity $(Z=2.36, p=0.02, O R=2.9795 \% \mathrm{Cl}=1.28$ to 6.90$)$; the lack of support from their family is another barrier in relation to their low physical activity $(Z=2.91, p=0.003, O R=3.82$, $95 \% \mathrm{Cl}=161$ to 9.10 ); another barrier was the lack of places (such as health centers and gyms for older adults) to perform physical activity $(Z=4.2, p<0.05, O R=5.8,95 \% \mathrm{Cl}=2.5$ to 13.3$)$.

Conclusion. The nursing designing programs to perform physical activity in older adults should take into account these perceived barriers.

\section{INTRODUCCIÓN}

Actualmente, el ser humano tiene mayor esperanza de vida debido a los avances en ciencia y tecnología de la salud; la Organización der las Naciones Unidas (ONU) ha considerado que en los siguientes 20 años, la población de adultos mayores en Latinoamérica se incrementará en un $4.5>\%{ }^{1}$. Este efecto demográfico afecta y afectará a México. Actualmente, en este país, hay 106.7 millones de habitantes y de acuerdo a las proyecciones del Consejo Nacional de Población (CoNaPo), los adultos mayores son 9'490, 250 de habitantes, representando el $8.8 \%{ }^{2,3}$. El estado de Aguascalientes tiene 1'141, 946 de habitantes, siendo los adultos mayores el $7.1 \%$ de la población ${ }^{4}$.

Un estilo de vida no saludable, tal como el sedentarismo, contribuye a padecer sobrepeso y obesidad, siendo factores de riesgo para enfermedades crónicas como diabetes, enfermedad cardiaca y cáncer, y existe una relación directa entre el nivel de actividad física (AF) en la infancia y AF en la vida adulta ${ }^{5}$.

Los adultos mayores que no realizan AF tienen elevada probabilidad de padecer enfermedades crónicas y aquellos que ya las padecen, presentan tendencia a empeorar y a presentar complicaciones, convirtiéndose en un problema de gran impacto para ellos ${ }^{6}$.

El fenómeno del sedentarismo puede deberse a barreras internas o externas; el modelo de Promoción de la Salud de la Dra. Nola J. Pender dice que las barreras percibidas, externas o internas, son obstáculos que impiden que el adulto mayor 
adopte un estilo de vida saludable; las barreras externas son estímulos ambientales e interacciones significativas, mientras que las barreras internas son aspectos físicos y emocionales ${ }^{7}$.

Se hizo una revisión sistemática de la literatura y encontramos que los estudios de AF se enfocan en los beneficios obtenidos por su implementación, pero no obtuvimos hallazgos acerca de las causas de ninguna o baja AF en los adultos mayores en México.

El objetivo de este estudio es detectar la relación entre las barreras percibidas, internas y externas, con el nivel bajo de AF en adultos mayores de Aguascalientes.

\section{MATERIALES Y MÉTODOS}

Es un estudio transversal analítico. Fue aprobado por el Comité de Investigación y Bioética de la División de Ciencias de la Salud e Ingenierías del Campus Celaya Salvatierra de la Universidad de Guanajuato.Todos los adultos mayores registrados en el Sistema de Desarrollo Integral Familiar (DIF) del estado de Aguascalientes, fue la población del estudio; todos fueron invitados a participar y sólo lo hicieron los que firmaron el consentimiento informado. Posteriormente respondieron la versión corta del Cuestionario Internacional de Actividad Física (IPAQ), en español ${ }^{8,9}$ el cual mide el nivel de AF.

La versión corta de este cuestionario maneja tres tipos de actividad específica en los últimos siete días: caminar (días de la actividad y minutos empleados cada día para esa actividad), actividades de moderada intensidad (días de la actividad y minutos empleados cada día) y actividades vigorosas (días de la actividad y minutos empleados cada día) ${ }^{10}$. Para conocer el nivel de AF, IPAQ usa 3.3 MET caminar y se multiplica por los minutos y luego por los días empleados para esa actividad; para las actividades moderadas, usa 4 MET y se multiplica por los minutos y por los días empleados; para las actividades vigorosas, usa 8 MET y se multiplica por los minutos y por los días empleados; estas personas se clasificaron como baja AF si tuvieron menos de $600 \mathrm{MET}$-minutos/semana, nivel moderado de AF, con 600 a $1499 \mathrm{MET}$ minutos/semana, y alto nivel de AF para 1500 o más MET-minutos/semana ${ }^{9}$

Posteriormente, se aplicó el cuestionario para identificar las barreras percibidas, de Sechrist et als. ${ }^{11}$; 14 preguntas se incluyen para identificar las barreras para realizar $\mathrm{AF}$; cada item, tiene cuatro posibles respuestas: fuertemente en desacuerdo, desacuerdo, de acuerdo y fuertemente de acuerdo; para identificar si era una barrera o no, las respuestas se clasificaron en Sí/NO; Sí cuando fuertemente de acuerdo o de acuerdo, y NO en desacuerdo o fuertemente en desacuerdo; se obtuvo una Kappa intra-obserador de 0.8 e inter-obserador de 0.95 en población mexicana.

También, se midió peso en $\mathrm{Kg}$, en una bascula con altímetro, con el sujeto sin zapatos; la estatura fue obtenida usando el altímetro de la bascula, estando de pie y se obtuvo en metros; se calculó el Índice de Masa Corporal (IMC) usando el peso en $\mathrm{Kg}$ dividido entre la estatura en metros al cuadrado, para conocer el status de IMC adecuado (24.9 o menor), sobrepeso (25 -29.9) u obeso (30 o mayor) ${ }^{12}$.

\section{Tamaño de muestra}

Esperando un $80 \%$ de bajo nivel de AF sin apoyo de la familia y $20 \%$ con nivel bajo de AF con apoyo familiar, el tamaño de muestra mínimo es de 15 adultos mayores en 
cada grupo, con $95 \%$ de precisión y $80 \%$ de poder, aunque estudiaremos a todos los que aceptaron participar (Epidat 3.1, 2006, Xunta de Galicia y Organización Panamericana de la Salud).

\section{Análisis estadístico}

Se utilizó estadística descriptiva para las variables de la muestra de sujetos; para probar nuestra hipótesis, se utilizó la prueba de $Z$ para dos proporciones independientes y valor de $\mathrm{p}$, para cada barrera y nivel bajo de actividad física; el valor de significancia estadística de $\mathrm{p}$, se fijó en 0.05 . También se calculó la Razón de Momios (RM) para conocer el efecto de las barreras percibidas sobre el nivel de actividad física, y se obtuvieron los intervalos de confianza al 95\% para conocer el posible valor de RM en la población de adultos mayores.

Para el análisis estadístico se utilizó, STATA 10.0® (Stata Corporation, College Station, Texas, USA).

\section{RESULTADOS}

La muestra estuvo integrada por 150 adultos mayores, registrados en el DIF de Aguascalientes; predominaron las mujeres con 108 (72\%), 107 (71.3\%) tenían entre 60 y 70 años de edad; 106 (70.7\%) estaban casadas, $102(68 \%)$ eran amas de casa; 84 presentaban sobrepeso (56\%) (Tabla I). 117 (78\%) tenían bajo nivel de actividad física y sólo $33(22 \%)$ la realizaban en forma moderada.

Tabla I. Variable sociodemográficas en adultos mayores, Aguascalientes, Ags. México $2010(\mathrm{n}=150)$

\begin{tabular}{|l|r|r|}
\hline \multicolumn{1}{|c|}{ Variables } & $\mathrm{N}$ & \multicolumn{1}{|c|}{$\%$} \\
\hline Género & 108 & 72.0 \\
Femenino & 42 & 28.0 \\
Masculino & & \\
\hline Grupo de edad & 107 & 71.3 \\
$60-70$ & 40 & 26.7 \\
$71-80$ & 3 & 2.0 \\
$81-90$ & & \\
\hline Estado civil & 11 & 7.4 \\
Soltero(a) & 106 & 70.7 \\
Casado(a) & 24 & 16.0 \\
Viudo(a) & 5 & 3.3 \\
Divorciado(a) & 2 & 1.3 \\
Separado(a) & 2 & 1.3 \\
Unión libre & 102 & \\
\hline Ocupación & 7 & 68.0 \\
Ama de casa & 6 & 4.7 \\
Retirado & 3 & 4.0 \\
Vigilante & 32 & 2.0 \\
Comerciante & & 21.3 \\
Otra & 42 & \\
\hline Estatus de sobrepeso/obesidad & 84 & 28.0 \\
Adecuado (BMl 24.9 or less) & 24 & 16.0 \\
Sobrepeso (BMl 25.0 - 29.9) & & \\
Obesidad (BMl 30.0 or higher) & & \\
\hline
\end{tabular}

Fuente: Cuestionario para identificar barreras percibidas 
A la mayoría de los adultos mayores les gusta el ejercicio, pero no hay diferencia entre proporciones y el nivel bajo de AF ( $p>0.05)$; la fatiga que causa AF es una barrera interna, ya que la diferencia de proporciones en adultos mayores con nivel bajo de AF con y sin fatiga por la AF es significativa $(p<0.05)$ y hay una efecto importante de la fatiga sobre nivel bajo de $\mathrm{AF}(\mathrm{OR}=4.55)$; obviamente, si el adulto mayor tiene una lesión física, no podrá realizar $A F(p=0.058)$; no hay diferencias entre preferir ver TV o leer y bajo nivel de $A F(p>0.05)$; si el adulto mayor no sabe que la AF es una prioridad, es una barrera interna para la realización de la $\operatorname{AF}(p<0.05)$; la falta de conocimiento de que tipo de AF realizar también es una barrera interna $(p<0.05)$ (Tabla II).

Tabla II. Barreras internas y nivel de actividad física en adultos mayores.

Aguascalientes, Ags. México, 2010 ( $n=150)$.

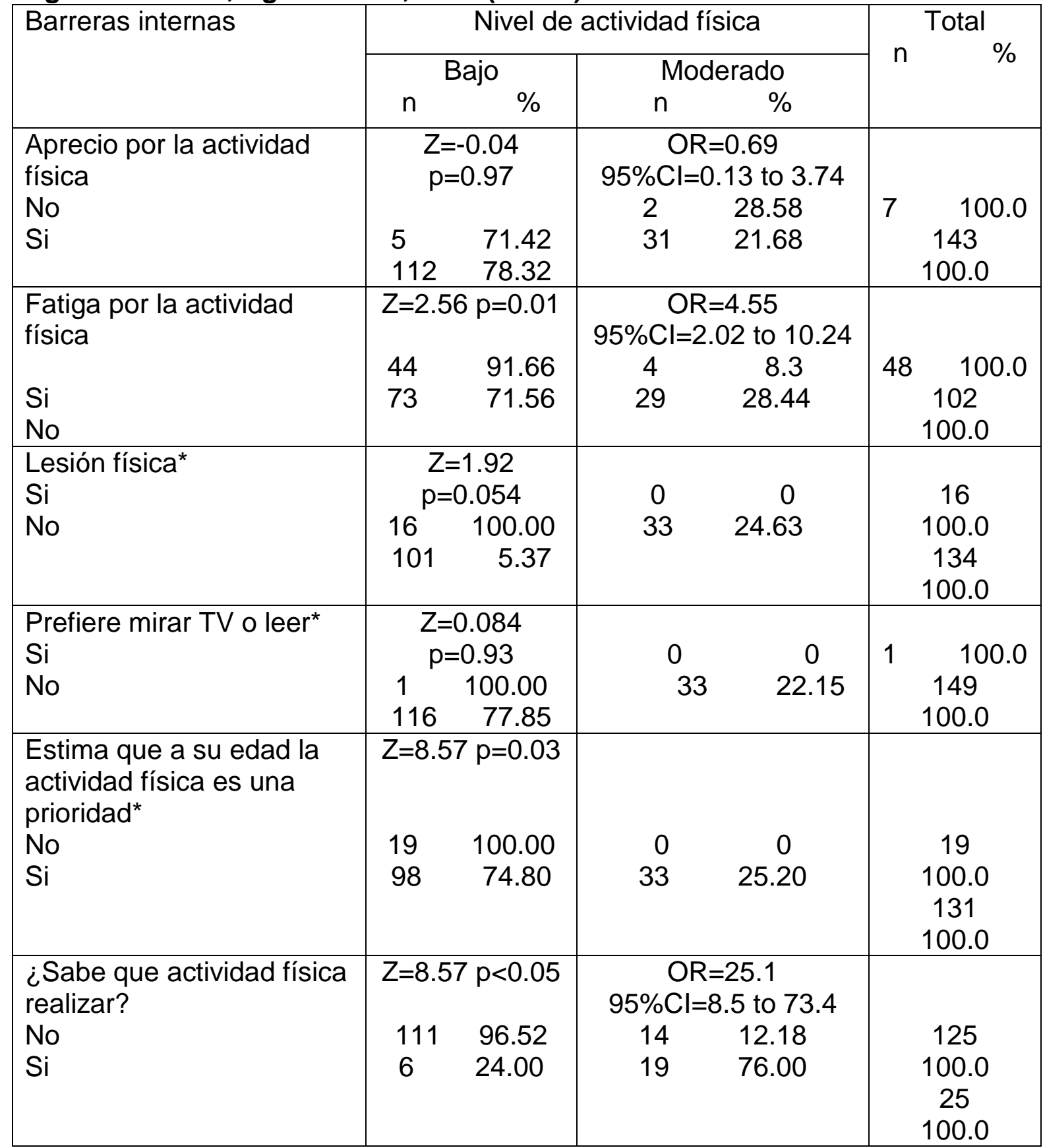

Fuente: Cuestionarios para identificar barreras percibidas e IPAQ 
* Razón de Momios e intervalos de confianza al 95\% no se pudieron calcular ya que una celda tiene 0 .

Los gastos por AF no son una barrera para 95\% de la muestra de adultos mayores y no está relacionada con nivel bajo de AF ( $p>0.05$ ), y para la mayoría de los sujetos en studio, el tiempo empleado para realizar AF no es una barrera $(p>0.05)$, la existencia de programas de AF en el vecindario no está relacionado con nivel bajo de actividad física ( $p>0.05)$; el uso de ropa deportiva es una barrera para realizar $A F(p<<0.05)$ y vivir solo(a) no ayuda a que el adulto mayor realice $A F(p=0.8)$; la familia del sujeto puede ser una barrera si no apoyan al adulto mayor $(p<0.05)$; otra barrera es la ausencia de espacios cercanos para AF $(p<0.05)$; si el adulto mayor no recibe información acerca de los beneficios de AF, también es una barrera $(p<0.05)($ Tabla III).

Tabla III. Barreras externas y nivel de actividad física en adultos mayors, Aguascalientes, Ags. $2010(n=150)$

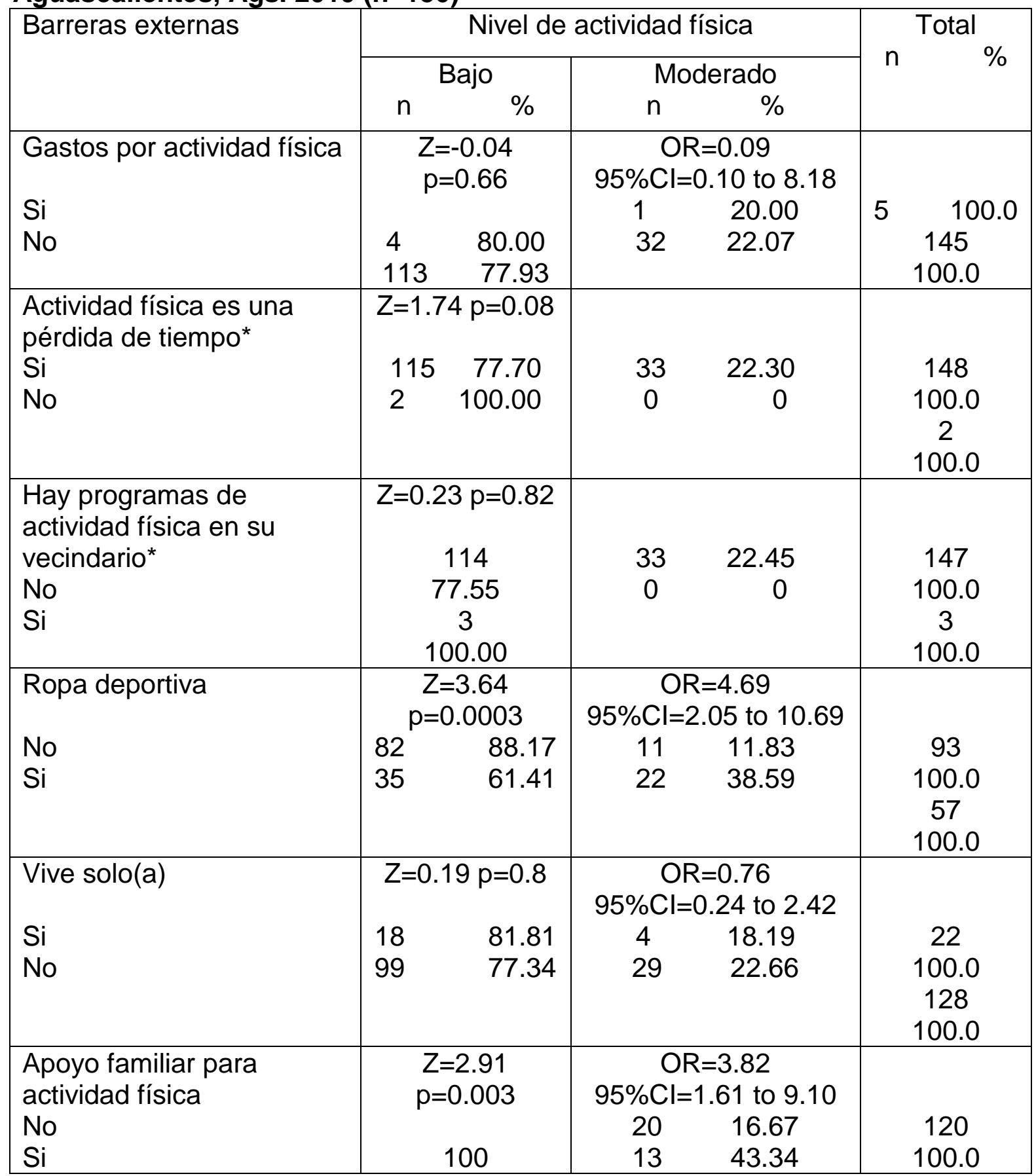




\begin{tabular}{|c|c|c|c|}
\hline & $\begin{array}{lr} & 83.33 \\
17 & 56.66\end{array}$ & & $\begin{array}{c}30 \\
100.0\end{array}$ \\
\hline $\begin{array}{l}\text { Sitios para realizar } \\
\text { actividad física cerca de } \\
\text { casa } \\
\text { No } \\
\mathrm{Si}\end{array}$ & $\begin{array}{c}Z=4.2 p<0.05 \\
90 \\
88.23 \\
27 \\
56.25\end{array}$ & \begin{tabular}{cc}
\multicolumn{2}{c}{$\mathrm{OR}=5.8$} \\
$95 \% \mathrm{Cl}=2.5$ to 13.3 \\
12 & 11.77 \\
21 & 43.75
\end{tabular} & $\begin{array}{c}102 \\
100.0 \\
48 \\
100.0\end{array}$ \\
\hline
\end{tabular}

Fuente: Cuestionario para identificar las barreras percibidas para actividad física e IPAQ

* Razón de Momios e intervalos de confianza al $95 \%$ no se pueden calcular ya que una celda tiene 0 .

\section{DISCUSIÓN}

La muestra fue obtenida por invitación para todos los adultos mayores registrados en el Sistema DIF y puede haber sesgo de selección. Es posible que haya sesgo de respuesta pero al usar la misma metodología en la aplicación de los cuestionarios fue utilizada para minimizarlo.

$80 \%$ de bajo nivel de AF sin apoyo familiar y $20 \%$ con apoyo familiar para la realización de actividad física se esperaba; $83.33 \%$ sin apoyo familiar y $56.66 \%$ con apoyo familiar fue encontrado en el estudio, y demostró que las proporciones de bajo nivel de actividad física fueron estadísticamente diferentes entre adultos mayores con y sin apoyo familiar (Tabla III).

Mora y col. encontraron que la AF en adultos mayores incrementa su autonomía y apoyo social y las barreras como la fatiga y el apoyo familiar tienen relación con el nivel de $\mathrm{AF}^{13}$; estos hallazgos también fueron encontrados en Aguascalientes (Tabla II y III).

Lago $^{14}$ reportó que los adultos mayores que participan en programas de AF, incrementan su calidad de vida, disminuye la severidad de sus enfermedades; los resultados en este estudio, corroboran lo anterior, ya que el no tener donde realizar AF en sus comunidades o cerca de su casa, fue una de las barreras detectadas (Tabla III).

Aspectos importantes deben tomarse en cuenta, para establecer un plan de acción para contribuir y fortalecer conductas promotoras de salud, formando el modelo de promoción de la salud.

Dergance y col. estudiaron adultos mayores México-Americanos y EuropeosAmericanos, y encontraron que las principales barreras fueron ausencia de interés en $A F$, falta de compañía para realizar AF, no divertirse mientras realiza AF y ausencia de conocimiento acerca de los beneficios de la AF; estas barreras fueron más frecuentes en Europeos-Americanos que en los Mecxico-Americanos ${ }^{15}$. En los adultos mayores de Aguascalientes, las barreras percibidas fueron fatiga, incapacidad física, no considerar a la AF una prioridad, ausencia de conocimiento de que tipo de actividad física realizar, no tener ropa deportiva adecuada, falta de apoyo familiar y no haber sitios para realizar AF cercanos; estas barreras fueron similares a las reportadas por Deregance y col. 
Trost y col., reportaron falta de tiempo, cansancio, debilidad, temor a caídas o a lesionarse, clima inconveniente, ausencia de lugares para realizar AF y parejas que no realizan ejercicio, resultaron en una fuerte influencia contraria a la realización de $\mathrm{AF}^{16}$.

Podemos observar que hay una relación entre las barreras internas y externas que los adultos mayores perciben y el bajo nivel de actividad física, y esto incrementa su vulnerabilidad a las enfermedades y a sus complicaciones.

Booth y col., ${ }^{17}$ reportaron que las barreras más frecuentemente percibidas para realizar actividad física entre australianos, fueron tiempo insuficiente, falta de motivación, lesión o salud deficiente. En la muestra de adultos mayores de Aguascalientes, se encontró a la lesión y falta de apoyo familiar como barreras percibidas.

Los resultados de esta investigación mostraron que en adultos mayores existen barreras internas y externas que impiden realizar un nivel moderado o vigoroso de actividad física; entre las barreras internas, el apoyo familiar y la fatiga física son las que mostraron mayor relación con nivel de actividad física bajo; entre las barreras externas, la ausencia de sitios para realiza AF, cercanos a sus casas, programas específicos de AF para adultos mayores, ropa adecuada para AF y ausencia de información acerca de los beneficios de la actividad física para la salud. Demostramos que hay una relación entre algunas barreras internas y externas y el nivel de actividad física bajo en adultos mayores.

\section{CONCLUSIÓN}

Hay barreras percibidas por los adultos mayores que tienen relación con su nivel de realización de actividad física. Estas barreras deben tomarse en cuenta, cuando se diseñen programas para incrementar el nivel de actividad física en adultos mayores.

\section{REFERENCIAS}

1.-Salgado VN, Wong R. Género y pobreza: los determinantes de salud en edad adulta. Rev Salud Publica de Mex, 2007; 49(Supl.4):S515-S521.

2.- Consejo Mexicano de Población. Indice demográfico en adultos mayores. http://www.conapo.gob.mx/index.php?option=com content\&view=article\&id=70:enveje cimiento\&catid=25:que-es-conapo\&ltemid=228) (Accesado Nov/16/2009).

3.- Partida BV. Proyección de la población en México 2005-2050. Consejo Mexicano de Población, 2006 (http://www.conapo.gob.mx) (Acceso Nov /16/2009).

4.- Consejo Mexicano de Población. El envejecimiento de la población en México. (http://www.conapo.gob.mx) (Acceso Ago/14/2009).

5.- Becerra $M$, Diaz $H$. Nivel de actividad física: beneficios, barreras y eficacia en empleados oficiales. Avances de Enfermería, 2008; 26(2):43-50.

6.- Marquez S, Rodriguez J, De Abajo S. Sedentarismo y salud: efectos beneficiosos de la actividad física (Primer Trimestre). Apuntes de física y deportes, 2006.

7.- Pender NJ, Murdaugh CL, Curas MA. Health promotion in nursing practice. New Jersey, 4a ed. Prentice Hall, 2002.

8.- International Physical Activity Questionnaire (http://www.ipaq.ki.se) (Acceso Nov/11/2009). 
9.- Craig CL, Marshall AL, Sjöström M, Bauman AE, Booth ML, Ainsworth BE, et als. International physical activity questionnaire: 12-country reliability and validity. Med Sci Sports Exerc, 2003; 35(8):1381-95.

10.- Patterson E. Guidelines for data processing and analysis of the international physical activity questionnaire (IPAQ)-Short and long forms- Nov, 2005 (https://sites.google.com/site/theipaq/scoring-protocol) (Acceso Marzo 6, 2012).

11.- Sechrist KR, Walker SN, Pender NJ. Development and psychometric evaluation of the exercise benefits/barriers scale. Research in Nursing \& Health, 1987;10(6):357265.

12.- Assesing your weight and health risk. Aim for a healthy weight. National Heart Lung and Blood Institute.

(http://www.nhlbi.nih.gov/health/public/heart/obesity/lose wt/risk.htm )

(Acceso Abril 12, 2012).

13.- Mora M, Araya G, Ozols A. Perspectiva subjetiva de calidad de vida en el adulto mayor, diferencias relacionadas a actividad física y género. Revista MHSalud, 2004; $1(1)$.

(http://www.revistas.una.ac.cr/index.php/mhsalud/article/view/312) (Acceso Feb /14/ 2012).

14.- Lago J. Prescripción de programas de ejercicio para adultos mayores en $\begin{array}{llll}\text { gimnasios. } & \text { Revista } & \text { digital, } & \text { 2005; }\end{array}$ (http://www.efdeportes.com/efd81/fitness.htm) (Acceso Feb/14/2012).

15.- Dergance J, Calmbach WL, Dhanda R, Miles TP, Hazuda HP, Mouton CP. Barriers to and benefits of leisure time physical activity in the elderly: across cultures. Journal of the American Geriatrics Society, 2003; 51(6):863-8.

16.- Trost SG, Owen N, Bauman AE, Sallis JF, Brown W. Correlates of adults' participation in physical activity: review and update. Med Sci Sports Exerc, 2002; 34(12): 1996-2001.

17.- Booth ML, Bauman A, Owen N, Gore CJ. Physical activity preferences, preferred sources of assistance, and perceived barriers to increased activity among physically inactive Australians. Preventive Medicine, 1997;26(1):131-7. 